\title{
Risk Factors for Posterior Cage Migration after Lumbar Interbody Fusion Surgery
}

\author{
Dong-Yeong Lee ${ }^{1}$, Young-Jin Park ${ }^{2}$, Sang-Youn Song ${ }^{2}$, Soon-Taek Jeong ${ }^{2}$, Dong-Hee Kim ${ }^{2}$ \\ ${ }^{1}$ Department of Orthopaedic Surgery, The Armed Forces Daegu Hospital, Gyeongsan, Korea \\ ${ }^{2}$ Department of Orthopaedic Surgery, Research Institute of Clinical Medicine, Institute of Health Sciences, Gyeongsang National University Hospital, \\ Gyeongsang National University School of Medicine, Jinju, Korea
}

Study Design: A retrospective clinical case series.

Purpose: To determine the strength of association between cage retropulsion and its related factors.

Overview of Literature: Lumbar interbody fusion with cage can obtain a firm union and can restore the disc height with normal sagittal and coronal alignment. Although lumbar interbody fusion procedures have satisfactory clinical outcomes, peri- and postoperative complications regarding the cage remain challenging.

Methods: From January 2006 to June 2016, 1,047 patients with lumbar degenerative disc disease who underwent posterior lumbar interbody fusion or transforaminal interbody fusion at Gyeongsang National University Hospital were enrolled. Medical records and pre- and postoperative radiographs were reviewed to identify significant cage retropulsion-related factors. The associations between cage retropulsion with various risk factors were evaluated by calculating odds ratios (ORs) and 95\% confidence intervals (Cls) using multiple logistic regression analysis.

Results: 0f 1,229 disc levels, 16 cases (1.3\%, 10 men and 6 women) had cage retropulsion. Univariate analysis revealed no significant differences between the cage retropulsion group and the no cage retropulsion group with regard to demographic data such as age, sex, weight, height, body mass index (BMI), smoking habits, presence of osteoporosis, and duration of follow-up. Multivariate analysis revealed that low BMI (OR, 0.875; $95 \% \mathrm{Cl}, 0.771-0.994 ; p=0.040)$, presence of screw loosening (OR, 27.400; $95 \% \mathrm{Cl}, 7.818-$ 96.033; $p<0.001)$, and pear-shaped disc (OR, 9.158; 95\% Cl, 2.455-34.160; $p=0.001)$ were significantly associated with cage retropulsion.

Conclusions: This study demonstrated that low BMI, loosening of posterior instrumentation, and pear-shaped disc were associated with cage retropulsion after lumbar interbody fusion. Therefore, when performing lumbar interbody fusion with a cage, surgeons should have skillful surgical techniques for firm fixation to prevent cage retropulsion, particularly in non-obese patients.

Keywords: Spine; Lumbar vertebrae; Spinal fusion; Complications

\section{Introduction}

With the increase in the aged population, the incidence of degenerative lumbar disease is also increasing. Posterior lumbar interbody fusion (PLIF) or transforaminal LIF (TLIF) is widely used for treating degenerative lumbar diseases such as degenerative spondylolisthesis, degenerative disc disease, and degenerative lumbar scoliosis [1-

Received Mar 15, 2017; Revised May 5, 2017; Accepted May 29, 2017

Corresponding author: Dong-Hee Kim

Department of Orthopaedic Surgery, Research Institute of Clinical Medicine, Institute of Health Sciences, Gyeongsang National University School of Medicine, 15 Jinju-daero 816beon-gil, Jinju 52727, Korea

Tel: +82-55-750-8669, Fax: +82-55-761-9477, E-mail: dhkim8311@gnu.ac.kr 
7]. When surgeons perform interbody fusion to promote spinal interbody union, a fusion cage packed with autocancellous bone or allo-bone is usually inserted into the disc space after vertebral endplate decortication. LIF with a cage can obtain a firm union and can restore the disc height with normal sagittal and coronal alignment. In addition, the spinal nerve root or compressed dural sac can be secondarily restored by increasing the disc height. Although these procedures have satisfactory clinical outcomes, peri- and postoperative complications remain challenging problems [5,8-10].

Several studies have reported regarding cage-related complications such as subsidence, migration, and mechanical failure [11-14]. Although many studies of cage subsidence or mechanical failure of cages after PLIF or TLIF have been published, few have evaluated the risk factors for cage retropulsion, a complication that can result in narrowing of the spinal canal or foraminal stenosis. Cage retropulsion can even directly compress the dural sac or nerve root and cause neurological symptoms. To improve clinical symptoms, the protruded cage has to be theoretically removed, thus requiring a revision surgery. However, a revision surgery is technically demanding because of the massive fibrosis of adjacent tissues. In addition, vague clinical symptoms sometimes may be concerning for spine surgeons, making it difficult to decide a revision surgery.

In previous studies, various risk factors that affect cage retropulsion after LIF have been reported [15-17]. Brodke et al. [18] and Lund et al. [19] suggested that additional posterior instrumentation is critical for preventing cage retropulsion, particularly in terms of flexion-extension torque. Therefore, LIF using a cage alone is no longer recommended. In the same context, Uzi et al. [20] reported that cage retropulsion can occur during flexion movement and thus suggested that this could be prevented by additional posterior instrumentation. In addition, Kimura et al. [15] found that the risk factors for cage retropulsion after PLIF were a wide disc space with instability, multilevel fusion surgery, involvement of L5-S1, and pear-shaped disc space on lateral radiographs. Other studies $[16,21]$ have also suggested that higher posterior disc height $(\mathrm{PDH})$, presence of scoliotic curvature at anteroposterior (AP) view, undersized fusion cages, cage positioning, and cage type are possible risk factors for cage retropulsion. Although previous studies have evaluated the effect of a single risk factor on cage retropulsion, the effects of multiple factors have not been simultaneously investigated [15-
17]. In addition, no study has evaluated the strength of associations between various factors and the occurrence of cage retropulsion. We hypothesized that among various risk factors, loosening of posterior instrumentation and a disc shape were significantly associated with the occurrence of cage retropulsion after LIF. Therefore, this study aimed to determine the strength of associations between cage retropulsion and its related factors.

\section{Materials and Methods}

\section{Demographic data}

This study was approved by the Institutional Review Board of Gyeongsang National University Hospital (GNUH IRB no., 2017-02-007). From January 2006 to June 2016, 1,047 patients with lumbar degenerative disc disease who underwent PLIF or TLIF at our institution were retrospectively enrolled. The inclusion criteria were (1) patients aged $>20$ years, (2) those who underwent interbody fusion with posterior instrumentation for treating degenerative lumbar disease, and (3) availability of preand postoperative radiographs and medical records. The exclusion criteria were (1) patients who underwent interbody fusion surgery using stand-alone cage; (2) those who underwent interbody fusion using an expandable cage or allo-strut bone graft; (3) those who underwent anterior, direct lateral, or oblique lateral interbody fusion; and (4) those whose medical records were unavailable because of death, loss of follow-up, or absence of data. Finally, 744 patients ( 1,229 disc levels) were included. Of the 744 patients, 307 were males and 437 were females; their mean age, weight, height, and body mass index (BMI) were $64.4 \pm 9.6$ years, $61.1 \pm 10.6 \mathrm{~kg}, 158.7 \pm 9.2 \mathrm{~cm}$, and $24.2 \pm 3.4$ $\mathrm{kg} / \mathrm{m}^{2}$, respectively. The patients were followed up for 31.1 months (range, 1-129 months) postoperatively. Data such as smoking habits, presence of osteoporosis (T score on dual-energy X-ray absorptiometry), and symptoms after surgery were reviewed using medical records. The included patients were then classified into two groups: the cage retropulsion group and the no cage retropulsion group. The demographic characteristics of each group are summarized in Table 1.

\section{Radiological evaluation}

Pre- and postoperative radiographs were obtained and 
Table 1. Demographic data of the patients who underwent posterior lumbar interbody fusion

\begin{tabular}{lccc} 
Characteristic & Cage retropulsion $(\mathrm{N}=16)$ & No cage retropulsion $(\mathrm{N}=728)$ & $p$-value \\
Age $(\mathrm{yr})$ & $68.3 \pm 7.8$ & $64.3 \pm 9.6$ & 0.104 \\
Sex & 10 & & 0.081 \\
$\quad$ Male & 6 & 431 & \\
$\quad$ Female & $59.8 \pm 11.5$ & $61.2 \pm 10.6$ & 0.616 \\
Weight $(\mathrm{kg})$ & $162.3 \pm 10.4$ & $158.6 \pm 9.2$ & 0.245 \\
\hline Height $(\mathrm{cm})$ & $22.8 \pm 2.8$ & $24.3 \pm 3.4$ & 0.090 \\
\hline Body mass index $\left(\mathrm{kg} / \mathrm{m}^{2}\right)$ & 1 & 127 & 0.331 \\
Smoking & 0 & $31.0 \pm 28.6$ & 0.392 \\
\hline Osteoporosis & $32.1 \pm 17.9$ & 0.882 \\
\hline Follow-up (mo) & & & \\
\hline
\end{tabular}

Values are presented as mean \pm standard deviation or number.
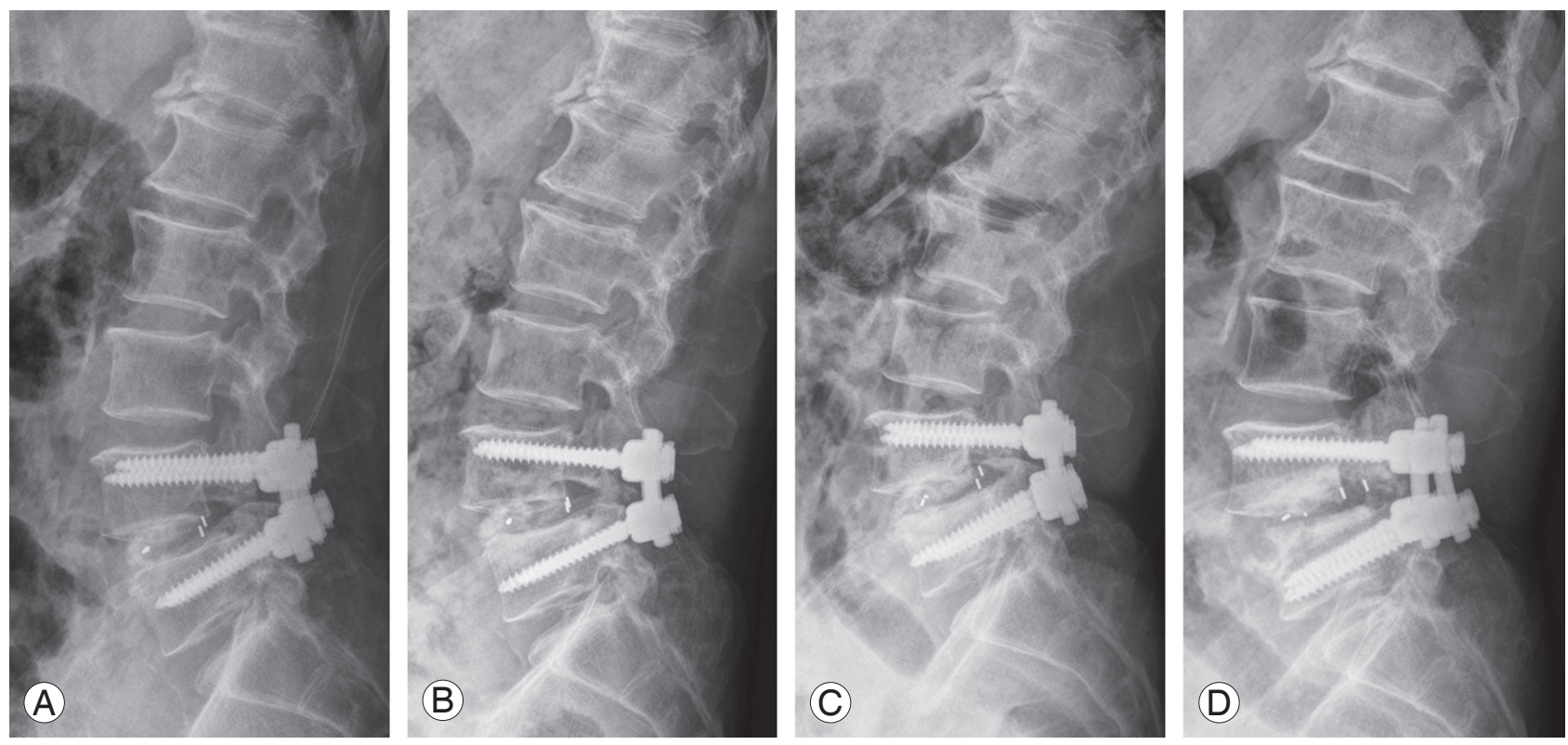

Fig. 1. Lateral lumbar radiographs showing the serial order of cage retropulsion after lumbar interbody fusion. (A) Postoperative day 1, (B) postoperative 1 month, (C) postoperative 6 months, and (D) postoperative 18 months.

evaluated using a Picture Archiving and Communications System. Cage retropulsion of the fusion cage was defined as the movement of the posterior margin of the cage beyond the posterior margin of either adjacent vertebral body (Fig. 1). Using AP and lateral simple radiographs, various factors such as fusion level, number of fusion segments, lumbar curvature, single- or multilevel fusion, presence of screw loosening, and cage type (polyether-ether-ketone [PEEK] or titanium) were evaluated (Table 2). In addition, disc shape (not pear-shaped or pear-shaped) (Fig. 2), presence of listhesis, degree of disc distraction, preoperative Cobb's angle at AP view, preand postoperative anterior disc height $(\mathrm{ADH}), \mathrm{PDH}$, and mean disc height $(\mathrm{MDH})$ were measured in the two groups (Table 3 ). On the basis of a previous study's definition [15], "pear-shaped disc" was defined as a disc with a convex surface in the posterior halves of the superior and inferior endplates with a concave surface in the anterior halves. $\mathrm{MDH}$ was calculated as the mean value of $\mathrm{ADH}$ and $\mathrm{PDH}$ (Fig. 3). The degree of disc distraction was investigated by assessing the differences in preoperative $\mathrm{MDH}$ and postoperative day $1 \mathrm{MDH}$ using lateral 
Table 2. Radiologic evaluation of the included patients

\begin{tabular}{|c|c|c|c|}
\hline Variable & Cage retropulsion & No cage retropulsion & $p$-value \\
\hline Fusion level & & & 0.268 \\
\hline $\mathrm{L} 1-\mathrm{L} 2$ & 0 & 20 & \\
\hline L2-L3 & 1 & 87 & \\
\hline L3-L4 & 1 & 275 & \\
\hline L4-L5 & 12 & 577 & \\
\hline L5-S1 & 2 & 254 & \\
\hline Fusion segments & $1.7 \pm 0.8$ & $1.7 \pm 0.8$ & 0.859 \\
\hline Curvature & & & 0.081 \\
\hline Lordosis & 11 & 441 & \\
\hline Straight & 3 & 262 & \\
\hline Kyphosis & 2 & 25 & \\
\hline Multi-level fusion & & & 0.853 \\
\hline Single & 8 & 381 & \\
\hline Multiple ( $\geq 2)$ & 8 & 347 & \\
\hline Screw loosening & 6 & 18 & $<0.001$ \\
\hline Cage type & & & 0.933 \\
\hline Poly-ether-ether-ketone & 5 & 391 & \\
\hline Titanium & 11 & 822 & \\
\hline
\end{tabular}

Values are presented as number or mean \pm standard deviation.

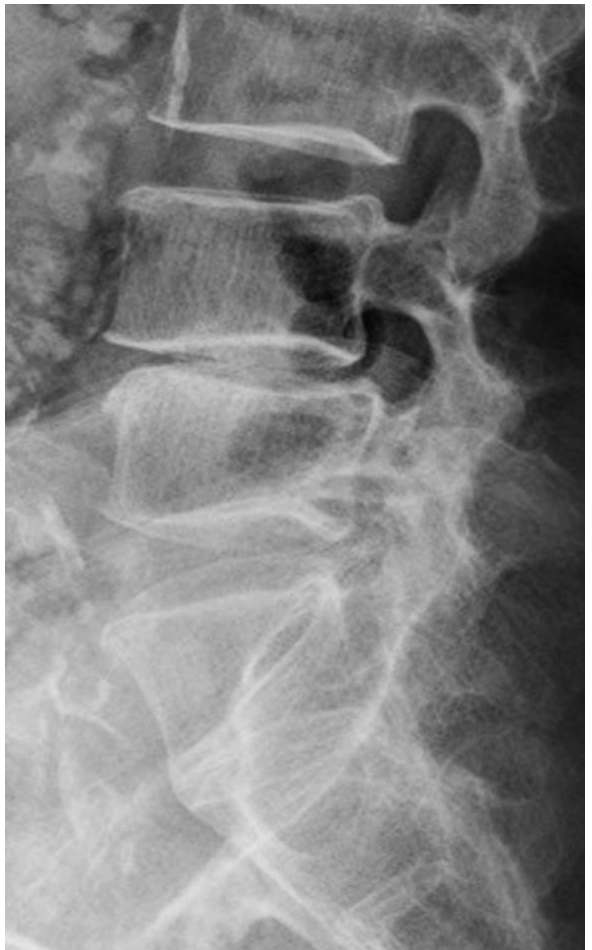

Fig. 2. Simple radiograph of a pear-shaped disc at the L4-L5 disc level. The disc with a convex surface in the posterior halves of the superior and inferior end plates and a concave surface in the anterior halves is shown. radiographs. To reduce the magnification of measurement errors, the measured disc height values were normalized using the sagittal diameter of the vertebral body between the midpoints of the anterior and posterior surface.

\section{Statistical analysis}

Differences between the two groups with regard to patient characteristics, fusion level, number of fusion segments, lumbar curvature, number of multilevel fusions, presence of screw loosening, and cage type were analyzed. In addition, differences between the two groups with respect to disc shape; presence of listhesis; pre- and postoperative $\mathrm{ADH}, \mathrm{PDH}$, and $\mathrm{MDH}$; degree of disc distraction; and preoperative Cobb's angle at AP view were evaluated. The reliability of measurements was assessed by examining the intra- or interobserver correlation coefficient. Two experienced orthopedic surgeons independently evaluated all measurements. Parameters were measured twice with a 4 -week interval between measurements by each surgeon. Independent $t$-tests were used for the comparison of numerical variables, and chi-square or Fisher's exact test was used for the comparison of categorical variables. The asso- 
Table 3. Radiologic evaluation of the disc space preparation

\begin{tabular}{|c|c|c|c|}
\hline Variable & Cage retropulsion ( $\mathrm{N}=16)$ & No cage retropulsion $(\mathrm{N}=1,213)$ & $p$-value \\
\hline Disc shape & & & $<0.001$ \\
\hline No pear-shaped & 10 & 1159 & \\
\hline Pear-shaped & 6 & 54 & \\
\hline Listhesis & 6 & 10 & 0.149 \\
\hline Preoperative ADH (mm) & $16.3 \pm 4.2$ & $15.3 \pm 4.0$ & 0.333 \\
\hline Preoperative PDH (mm) & $9.5 \pm 2.6$ & $8.9 \pm 4.1$ & 0.592 \\
\hline Preoperative MDH (mm) & $12.9 \pm 2.8$ & $12.1 \pm 3.2$ & 0.346 \\
\hline Postoperative ADH (mm) & $16.1 \pm 4.1$ & $16.2 \pm 3.9$ & 0.915 \\
\hline Postoperative PDH (mm) & $12.0 \pm 3.5$ & $10.2 \pm 3.0$ & 0.020 \\
\hline Postoperative MDH (mm) & $14.5 \pm 2.1$ & $13.2 \pm 2.8$ & 0.072 \\
\hline Disc distraction (mm) & $1.6 \pm 2.4$ & $1.1 \pm 3.3$ & 0.543 \\
\hline Preoperative Cobb's angle at anteroposterior view $\left({ }^{\circ}\right)$ & $2.4 \pm 2.4$ & $2.5 \pm 2.5$ & 0.950 \\
\hline
\end{tabular}

Values are presented as number or mean \pm standard deviation.

$\mathrm{ADH}$, anterior disc height; $\mathrm{PDH}$, posterior disc height; $\mathrm{MDH}$, mean disc height.

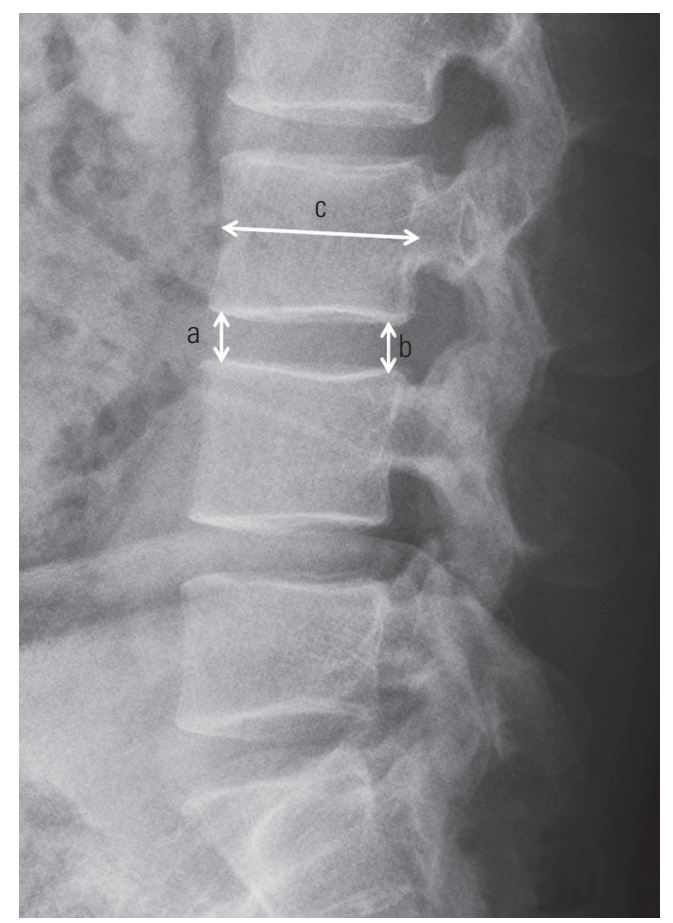

Fig. 3. Measurement of pre- and postoperative disc heights in the lumbar lateral view. The mean disc height was calculated as $(a+b) / 2$. a, anterior disc height; b, posterior disc height; $c$, sagittal diameter of the vertebral body measured between the midpoints of the anterior and posterior surfaces.

ciations of cage retropulsion with various risk factors were evaluated by calculating the odds ratios (ORs) and 95\% confidence intervals (CIs) using multiple logistic regres- sion analysis. First, univariate logistic regression analyses were performed for all variables, followed by multiple logistic regression analysis using only variables with $p$ values of $<0.2$, which were obtained from the univariate analyses. A multivariate logistic regression analysis was performed after assessing multicollinearity using the variance inflation factor (VIF) and conditional index. Variables with VIF of $<10$ and a conditional index of $<10$ were considered to indicate the absence of multicollinearity [22]. The Hosmer-Lemeshow test was used to determine the goodness of fit for the multivariate logistic regression model. All analyses were performed using the PASW SPSS software program ver. 18.0 (SPSS Inc., Chicago, IL, USA). Statistical significance was set at $p$-values of $<0.05$. The significance of the Hosmer-Lemeshow test was set at $p$-values of $>0.05$.

\section{Results}

\section{Study populations}

The intra- and interobserver reliabilities of measurements were 0.902 and 0.884 , respectively. The reliability of measurements was relatively high in this study. Of 1,229 disc levels, 16 cases (1.3\%, 10 men and 6 women) had cage retropulsion, and the mean onset time of cage retropulsion was 8.3 months (range, 1-48 months). The average age of the patients was $68.3 \pm 7.8$ years. Although 355 patients 
Table 4. Characteristics of the patients who developed cage retropulsion $(\mathrm{N}=16)$

\begin{tabular}{|c|c|c|c|c|c|c|c|c|c|c|c|}
\hline Case & $\begin{array}{l}\text { Age } \\
\text { (yr) }\end{array}$ & Sex & Diagnosis & $\begin{array}{l}\text { Fusion } \\
\text { level }\end{array}$ & $\begin{array}{l}\text { Fusion } \\
\text { type }\end{array}$ & $\begin{array}{c}\text { CR } \\
\text { level }\end{array}$ & Cage & $\begin{array}{c}\text { Fixation } \\
\text { type }\end{array}$ & $\begin{array}{c}\text { Screw } \\
\text { loosening }\end{array}$ & $\begin{array}{l}\text { CR time } \\
\text { (mo) }\end{array}$ & Symptom after CR \\
\hline 1 & 69 & M & $\mathrm{DSL}$ & L4-L5 & PLIF & L4/L5 & PEEK & Bilateral & No & 2 & No \\
\hline 2 & 76 & $\mathrm{~F}$ & SS & Ł4- L5 & PLIF & L4/L5 & PEEK & Bilateral & No & 5 & LBP with radiculopathy \\
\hline 3 & 63 & M & SS & L4-L5 & PLIF & L4/L5 & PEEK & Bilateral & No & 48 & No \\
\hline 4 & 66 & M & SS & L4-L5 & PLIF & L4/L5 & PEEK & Bilateral & Yes & 1 & LBP without radiculopathy \\
\hline 5 & 70 & $\mathrm{~F}$ & DSL & L3-L5 & PLIF & L4/L5 & PEEK & Bilateral & Yes & 2 & No \\
\hline 6 & 78 & $\mathrm{~F}$ & SS & L4-S1 & TLIF & L4/L5 & PEEK & Bilateral & Yes & 1 & No \\
\hline 7 & 69 & $\mathrm{M}$ & DSL & L3-S1 & PLIF & L5/S1 & Titanium & Bilateral & Yes & 3 & LBP \\
\hline 8 & 71 & M & DS & L3-S1 & PLIF & $\mathrm{L} 5 / \mathrm{S} 1$ & Titanium & Bilateral & Yes & 1 & No \\
\hline 9 & 77 & $M$ & SS & L4-S1 & TLIF & L4/L5 & PEEK & Bilateral & Yes & 1 & Radiculopathy \\
\hline 10 & 73 & M & DSL & L4-L5 & PLIF & $L 4 / L 5$ & Titanium & Bilateral & No & 48 & LBP \\
\hline 11 & 71 & $M$ & SS & L2-L4 & PLIF & L2/L3 & Titanium & Bilateral & No & 1 & LOM, L/E weakness \\
\hline 12 & 52 & M & SS & L3-L4 & PLIF & L3/L4 & PEEK & Bilateral & No & 7 & No \\
\hline 13 & 69 & $\mathrm{~F}$ & SS & L2-L5 & PLIF & L4/L5 & PEEK & Bilateral & No & 2 & Radiculopathy \\
\hline 14 & 54 & $\mathrm{~F}$ & DDD & L3-L5 & PLIF & $L 4 / L 5$ & Titanium & Bilateral & No & 2 & No \\
\hline 15 & 59 & $\mathrm{~F}$ & DSL & L4-L5 & PLIF & L4/L5 & PEEK & Bilateral & No & 1 & No \\
\hline 16 & 75 & M & SS & L4-L5 & PLIF & L4/L5 & PEEK & Bilateral & No & 7 & No \\
\hline
\end{tabular}

CR, cage retropulsion; $M$, male; F, female; DSL, degenerative spondylolisthesis; PLIF, posterior lumbar interbody fusion; PEEK, poly-ether-ether-ketone; SS, spinal stenosis; LBP, lower back pain; TLIF, transforaminal lumbar interbody fusion; DS, degenerative scoliosis; LOM, limitation of motion; $L / E$, lower extremity; DDD, degenerative disc disease.

underwent multilevel spinal fusion, there was no case of cage retropulsion simultaneously with $\geq 2$ segments in a single patient. Demographic data such as age, sex, weight, height, BMI, smoking habits, presence of osteoporosis, and duration of follow-up were not significantly different between the two groups. Among 16 patients with cage retropulsion, the diagnoses for these patients included degenerative spondylolisthesis $(n=5)$, lumbar spinal stenosis $(n=9)$, lumbar degenerative scoliosis $(n=1)$, and degenerative disc disease $(n=1)$. Fusion segments included L1-L2 ( 1 of 88 patients, $1.1 \%$ ), L3-L4 (1 of 276 patients, $0.4 \%$ ), L4-L5 (12 of 589 patients, $2.0 \%$ ), and L5-S1 (2 of 256 patients, $0.8 \%$ ). The mean fusion level was $1.7 \pm 0.8$ segments in these patients. Eight patients underwent single-level fusion, and eight patients underwent multilevel spinal fusion. Cages with posterior retropulsion were divided into PEEK (5 levels) and titanium (11 levels). Among the 16 patients with cage retropulsion, nine had no symptoms, six had lower back pain and/or radiculopathy, and one had motion limitations with lower extremity weakness. Although seven patients had clinical symptoms, none required a revision surgery. The detailed characteristics of the patients with cage retropulsion are summarized in
Table 4.

\section{Radiological evaluations}

Cage retropulsion was detected at a mean period of 8.3 months (range, 1-48 months) after surgery. Of the 16 patients, nine had no symptoms and seven had lower back pain and/or radiculopathy (Table 4). However, seven symptomatic patients could tolerate their symptoms without requiring a revision surgery. Six of the 16 patients had screw loosening, which was significantly $(p<0.001)$ associated with the presence of cage retropulsion. Among the 16 patients with cage retropulsion, six had pear-shaped discs, which were also significantly $(p<0.001)$ associated with cage retropulsion. Although six patients had spinal listhesis on preoperative radiographs, the presence of listhesis was not associated with cage retropulsion.

To evaluate the influence of disc height, pre- and postoperative $\mathrm{ADH}, \mathrm{PDH}$, and $\mathrm{MDH}$; degree of disc distraction; and Cobb's angle at AP view were compared between the migrated and non-migrated levels. The results showed that preoperative $\mathrm{ADH}, \mathrm{PDH}$, and $\mathrm{MDH}$; postoperative $\mathrm{ADH}$ and $\mathrm{MDH}$; degree of disc distraction; and Cobb's 
Table 5. Strengths of associations between various factors and cage retropulsion in the multivariate analyses

\begin{tabular}{lcr} 
Variable & Multivariate analysis & $p$-value \\
Low body mass index $\left(\mathrm{kg} / \mathrm{m}^{2}\right)$ & $0.875(0.771-0.994)$ & 0.040 \\
Screw loosening & $27.400(7.818-96.033)$ & $<0.001$ \\
Pear-shaped disc & $9.158(2.455-34.160)$ & 0.001 \\
\hline
\end{tabular}

Values are presented as odds ratio (95\% confidence interval).

angle at AP view were not significantly different between the two groups. However, postoperative PDH was significantly ( $p=0.020$ ) associated with cage retropulsion.

\section{Multivariate analysis of risk factor for cage retropulsion}

Regarding the multicollinearity evaluation among the significant variables determined by the univariate analyses, VIFs of all variables with $p$-values of $<0.2$ were $<10$. Thus, all variables with $p$-values of $<0.2$ were included in the multivariate logistic regression analysis. The $p$-value of the Hosmer-Lemeshow test was 0.626 , which indicated a good fit of data. In univariate analyses, variables with a $p$-value of $<0.2$ included age, sex, BMI, lumbar curvature, disc shape, presence of listhesis, postoperative $\mathrm{PDH}$, postoperative $\mathrm{MDH}$, and presence of screw loosening. Using these factors, multivariate analysis was performed and showed that only low BMI (OR, 0.875; 95\% CI, 0.7710.994; $p=0.040$ ), presence of screw loosening (OR, 27.400; 95\% CI, 7.818-96.033; $p<0.001)$, and pear-shaped disc (OR, 9.158; 95\% CI, 2.455-34.160; $p=0.001$ ) were significantly associated with cage retropulsion (Table 5).

\section{Discussion}

In this study, risk factors for cage retropulsion after LIF surgery were assessed by retrospectively analyzing clinical data. Previous studies on cage retropulsion have shown that the incidence of cage retropulsion ranged from $0.8 \%$ to $25 \%[15,17,21]$. Although the definition of cage retropulsion and study population was somewhat different in each study, our result (1.3\%) was consistent with that of previous studies. Based on the multivariate analysis, the most important finding of this study was that low BMI, loosening of posterior instrumentation, and disc shape were significantly associated with cage retropulsion after LIF, a finding that supported our study hypothesis.

In previous studies, various risk factors associated with cage retropulsion after interbody fusion have been reported. Kimura et al. [15] reported that cage retropulsion developed within 2 months after surgery in all cases. They identified the following risk factors: (1) involvement of L5/S1, (2) pear-shaped disc space, (3) wide disc space with instability, and (4) multilevel fusion surgery. To avoid the complication of cage retropulsion, they recommended the use of expandable cages. In addition, Abbushi et al. [21] reported that cage positioning and cage type influenced cage retropulsion. In particular, the medio-medial cage position and closed box cages appeared to be associated with higher cage retropulsion rates. Aoki et al. [16] suggested that a bullet-shaped cage, higher $\mathrm{PDH}$, presence of scoliotic curvature, and undersized fusion cages were possible risk factors for cage retropulsion. Although we did not perform analysis using all variables identified or suggested in previous studies because of insufficient data, we included as many factors as possible. We then performed multivariate analysis to determine the risk factors for cage retropulsion in our study. Our results also support those of previous studies.

Both univariate and multivariate analyses revealed that screw loosening of the posterior instrumentation was significantly associated with the occurrence of cage retropulsion. Some studies have revealed that the lack of posterior screw fixation was a main risk factor for cage retropulsion $[20,23]$. In settings with a lack of posterior instrumentation, cage retropulsion can occur because of spinal flexion forces, which leads to mechanical failure and intersegmental nonunion. Furthermore, a posteriorly migrated cage can compress the dural sac or spinal nerve root, leading to unsatisfactory clinical outcomes. In the same context, Brodke et al. [18] and Lund et al. [19] biomechanically assessed the initial stability of PLIF alone and with additional posterior fixation. Both studies suggested the importance of additional posterior instrumentation for preventing cage retropulsion. Applying posterior instrumentation and markedly increasing stiffness in axial compression can reduce the posterior bending force, particularly flexion-extension torque. Although it is unclear 
which factor between cage retropulsion and posterior instrumentation loosening precedes the other and because 10 of 16 patients with cage retropulsion did not present with loosening in posterior instrumentation, we assumed that insufficient posterior fixation leads to residual spinal instability, possibly causing posterior screw loosening and cage retropulsion. Thus, firm posterior instrumentation is critical for preventing cage retropulsion, which can lead to mechanical spinal failures.

In this study, the disc shape was classified into two groups on the basis of the study of Kimura et al. [15]: pear-shaped and non-pear-shaped discs. Regardless of the fused disc level, a pear-shaped disc was significantly associated with a higher incidence of cage retropulsion. Because a pear-shaped disc does not tend to make contact with all four corners of the cage in the sagittal plane, it may lead to instability between the endplate and cage. Although we could not investigate the cage size, Pan et al. [17] reported that the use of an undersized cage was potentially a risk factor for cage retropulsion. This mechanism might be associated with inadequate contact between the endplate and cage because the two studies mentioned above demonstrated that uneven stress generated by the cage and vertebral endplate could lead to instability. In addition, cage instability can be caused by excessive $\mathrm{PDH}$ distraction, which is a significant factor in univariate analysis. Although it was not a significant factor in our multivariate analysis, excessive PDH distraction may cause inadequate contact between the cage and vertebral endplate, possibly leading to cage retropulsion because of segmental instability; thus, PDH distraction could act as an independent risk factor for cage retropulsion. Therefore, when surgeons perform endplate preparation in patients with pear-shaped discs, meticulous endplate decortications are required to avoid cage retropulsion. Furthermore, an adequate cage size with sufficient axial compression is required for firm interbody fusion.

Our analysis showed that low BMI was also associated with cage retropulsion. In other words, patients with a higher BMI had a lower incidence of cage retropulsion. No previous study has reported that low BMI acted as a risk factor for cage retropulsion. A higher BMI might be associated with a higher axial compression force in the vertebral body. Aoki et al. [24] suggested that adequate compression needs to be applied during screw fixation to prevent cage migration. Pan et al. [17] suggested that bilateral pedicle screw fixation for PLIF procedure is more beneficial than unilateral pedicle screw fixation. In similar ways, a higher BMI might have contributed to the increase in axial compression between the cage and vertebral body. Thus, a higher BMI might act as a protective factor against cage retropulsion. To investigate this possibility, a wellcontrolled large-scale study is required.

Among the 16 patients with cage retropulsion, nine had no symptoms, six had lower back pain and/or radiculopathy, and one had motion limitation with lower extremity weakness. Although seven patients had clinical symptoms, none of these patients required a revision surgery. In previous studies, the incidence of a revision surgery for cage retropulsion has been reported to range from $33.3 \%$ to $75.0 \%[15,17]$. Revision surgery for cage retropulsion is technically challenging. Because of scar tissues and fibrosis of soft tissues, cage removal is a complicated procedure and can potentially increase postoperative pain and the probability of neurological deficits [24]. Pan et al. [17] suggested that a revision surgery is essential for patients with neurological deficits, whereas conservative treatment is recommended for asymptomatic patients. However, on the basis of our own and previous results, we do not believe that cage retropulsion will necessarily show clinical symptoms, and a revision surgery may not always be required. In contrast to the opinions of Pan et al. [17], neurological symptoms are not absolute indications for a surgery. Thus, for patients with tolerable symptoms, conservative treatment should be considered as a treatment option.

The average onset time of cage retropulsion was relatively early. In previous studies, the average onset time was reported to be $2.75, \leq 2$, or $\leq 3$ months [15-17]. Conversely, we found that the mean onset time of cage retropulsion was 8.3 months (range, 1-48 months). However, our study had some errors. Among the 16 patients with cage retropulsion, two were lost to follow-up. Because these two patients visited our institution again at 48 months after cage retropulsion was observed the first time, the onset time of cage retropulsion was unclear for these two patients. Hence, when we used the data of the remaining 14 patients, we found that the mean onset time of cage retropulsion was 2.6 months (range, 1-7 month), which was consistent with the results of previous studies. The current study and previous studies showed that cage retropulsion had an early onset time, implying that the occurrence of cage retropulsion might be closely associated with technical errors when initially attempting to achieve spinal sta- 
bility. To prevent this type of complication, stable fixation for interbody fusion is essential.

Our study had some limitations. First, this was a retrospective study. In addition, many cases were excluded because of insufficient data descriptions, which may have caused a selection bias. Second, a relatively small number of cage retropulsion cases were included in this analysis because of the low incidence, making it difficult to draw many meaningful conclusions. Third, different risk factors such as surgical techniques (PLIF or TLIF), cage position, cage type, and cage size that may have affected cage retropulsion were not considered in this study, which might have limited the conclusions. Although this was not a randomized, prospective, or case-controlled design, this was the first study to consider multifactorial risk factors for cage retropulsion after LIF. In the future, large-scale prospective studies that control for such independent factors through high-quality medical research are required.

\section{Conclusions}

In conclusion, this study demonstrated that low BMI, loosening of posterior instrumentation, and pear-shaped disc were associated with cage retropulsion after LIF. Therefore, when surgeons perform LIF with a cage, strong consideration should be given to applying skillful surgical techniques to achieve firm fixation to prevent cage retropulsion, particularly in non-obese patients.

\section{Conflict of Interest}

No potential conflict of interest relevant to this article was reported.

\section{References}

1. Agazzi S, Reverdin A, May D. Posterior lumbar interbody fusion with cages: an independent review of 71 cases. J Neurosurg 1999;91(2 Suppl):186-92.

2. Humphreys SC, Hodges SD, Patwardhan AG, Eck JC, Murphy RB, Covington LA. Comparison of posterior and transforaminal approaches to lumbar interbody fusion. Spine (Phila Pa 1976) 2001;26:567-71.

3. Lauber S, Schulte TL, Liljenqvist U, Halm H, Hackenberg L. Clinical and radiologic 2-4-year results of transforaminal lumbar interbody fusion in degenerative and isthmic spondylolisthesis grades 1 and 2 .
Spine (Phila Pa 1976) 2006;31:1693-8.

4. Lowe TG, Tahernia AD, O’Brien MF, Smith DA. Unilateral transforaminal posterior lumbar interbody fusion (TLIF): indications, technique, and 2-year results. J Spinal Disord Tech 2002;15:31-8.

5. Taneichi H, Suda K, Kajino T, Matsumura A, Moridaira H, Kaneda K. Unilateral transforaminal lumbar interbody fusion and bilateral anterior-column fixation with two Brantigan I/F cages per level: clinical outcomes during a minimum 2-year follow-up period. J Neurosurg Spine 2006;4:198-205.

6. Aygun H, Cakar A, Huseyinoglu N, Huseyinoglu $\mathrm{U}$, Celik R. Clinical and radiological comparison of posterolateral fusion and posterior interbody fusion techniques for multilevel lumbar spinal stabilization in manual workers. Asian Spine J 2014;8:571-80.

7. Pawar AY, Hughes AP, Sama AA, Girardi FP, Lebl DR, Cammisa FP. A comparative study of lateral lumbar interbody fusion and posterior lumbar interbody fusion in degenerative lumbar spondylolisthesis. Asian Spine J 2015;9:668-74.

8. Booth KC, Bridwell KH, Eisenberg BA, Baldus CR, Lenke LG. Minimum 5-year results of degenerative spondylolisthesis treated with decompression and instrumented posterior fusion. Spine (Phila Pa 1976) 1999;24:1721-7.

9. Boos N, Marchesi D, Zuber K, Aebi M. Treatment of severe spondylolisthesis by reduction and pedicular fixation. A 4-6-year follow-up study. Spine (Phila Pa 1976) 1993;18:1655-61.

10. Sudprasert W, Choovongkomol K, Piyapromdee U, Leownorasate M. Impact on neurological recovery of transforaminal debridement and interbody fusion versus transpedicular decompression in combination with pedicle screw instrumentation for treating thoracic and lumbar spinal tuberculosis. Asian Spine J 2016;10:543-52.

11. McAfee PC, Cunningham BW, Lee GA, et al. Revision strategies for salvaging or improving failed cylindrical cages. Spine (Phila Pa 1976) 1999;24:214753.

12. Tullberg T. Failure of a carbon fiber implant: a case report. Spine (Phila Pa 1976) 1998;23:1804-6.

13. Zhao FD, Yang W, Shan Z, et al. Cage migration after transforaminal lumbar interbody fusion and factors related to it. Orthop Surg 2012;4:227-32.

14. Smith AJ, Arginteanu M, Moore F, Steinberger A, 
Camins M. Increased incidence of cage migration and nonunion in instrumented transforaminal lumbar interbody fusion with bioabsorbable cages. J Neurosurg Spine 2010;13:388-93.

15. Kimura H, Shikata J, Odate S, Soeda T, Yamamura S. Risk factors for cage retropulsion after posterior lumbar interbody fusion: analysis of 1070 cases. Spine (Phila Pa 1976) 2012;37:1164-9.

16. Aoki Y, Yamagata M, Nakajima F, et al. Examining risk factors for posterior migration of fusion cages following transforaminal lumbar interbody fusion: a possible limitation of unilateral pedicle screw fixation. J Neurosurg Spine 2010;13:381-7.

17. Pan FM, Wang SJ, Yong ZY, Liu XM, Huang YF, Wu DS. Risk factors for cage retropulsion after lumbar interbody fusion surgery: series of cases and literature review. Int J Surg 2016;30:56-62.

18. Brodke DS, Dick JC, Kunz DN, McCabe R, Zdeblick TA. Posterior lumbar interbody fusion: a biomechanical comparison, including a new threaded cage. Spine (Phila Pa 1976) 1997;22:26-31.

19. Lund T, Oxland TR, Jost B, et al. Interbody cage stabilisation in the lumbar spine: biomechanical evaluation of cage design, posterior instrumentation and bone density. J Bone Joint Surg Br 1998;80:351-9.
20. Uzi EA, Dabby D, Tolessa E, Finkelstein JA. Early retropulsion of titanium-threaded cages after posterior lumbar interbody fusion: a report of two cases. Spine (Phila Pa 1976) 2001;26:1073-5.

21. Abbushi A, Cabraja M, Thomale UW, Woiciechowsky C, Kroppenstedt SN. The influence of cage positioning and cage type on cage migration and fusion rates in patients with monosegmental posterior lumbar interbody fusion and posterior fixation. Eur Spine J 2009;18:1621-8.

22. Belsley DA, Kuh E, Welsch RE. Regression diagnostics: identifying influential data and sources of collinearity. Hoboken: Wiley-Interscience; 2004.

23. Chen L, Yang H, Tang T. Cage migration in spondylolisthesis treated with posterior lumbar interbody fusion using BAK cages. Spine (Phila Pa 1976) 2005;30:2171-5.

24. Aoki Y, Yamagata M, Nakajima F, Ikeda Y, Takahashi K. Posterior migration of fusion cages in degenerative lumbar disease treated with transforaminal lumbar interbody fusion: a report of three patients. Spine (Phila Pa 1976) 2009;34:E54-8. 